\title{
Computer-aided analysis of Riemann sheet structures
}

\author{
André van Hameren* and Ronald Kleiss ${ }^{\dagger}$ \\ University of Nijmegen, Nijmegen, the Netherlands
}

November 17, 2018

\begin{abstract}
We report on experience with an investigation of the analytic structure of the solution of certain algebraic complex equations. In particular the behavior of their series expansions around the origin is discussed. The investigation imposes the need for an analysis of the singularities and the Riemann sheets of the solution, in which numerical methods are used.
\end{abstract}

*andrevh@sci.kun.nl

${ }^{\dagger}$ kleiss@sci.kun.nl 


\section{Introduction}

In various problems in theoretical physics we have encountered problems that are formulated as follows: consider an entire function $F$ of a complex number $y$, such that

$$
F(y) \sim y^{m} \quad \text { as } y \rightarrow 0
$$

with nonnegative integer $m$ (in practice, we have met cases with $m=1$ and $m=2$ ). The task at hand is then to find information about $y=y(x)$ such that

$$
F(y(x))=x^{m}
$$

In general, both the form of the series expansion of $y(x)$ around $x=0$ and the nature of its singularities are of interest. Such questions arise, for instance, in the combinatorial problem of determining the number of Feynman diagrams contributing to given scattering amplitudes in various quantum field theories [1], in the computation of the oscillation period of nontrivial one-dimensional potential wells [2], in the statistical bootstrap model for hot hadronic matter (refs. in [3] and for a recent discussion see [4]), and in renormalization theory connected with the 't Hooft transformation [5]. An important and interesting example, studied in detail in [3], is the so-called bootstrap equation:

$$
F_{B}(y)=2 y+1-e^{y},
$$

which obviously has $m=1$. We shall consider functions $F$ of the more general form

$$
F(y)=P(y)+e^{y} Q(y),
$$

where $P$ and $Q$ are polynomials of finite degree $d_{P}>0$ and $d_{Q} \geq 0$, respectively, with real coefficients. As our working example, taken from [2], we shall consider the function $F_{1}$ defined as

$$
F_{1}(y)=-2-2 y+2 e^{y},
$$

for which $m=2$. In this paper we shall concentrate on the analysis of the Riemann sheet structure of those solutions of these equations that have a series expansion around $x=0$. To determine the asymptotic behavior of these expansions, the nature of the singularities will be analyzed numerically. The results are justified by the fact that, in our calculations, only finite computer accuracy is required, as we shall demonstrate.

\section{Identification of the Riemann sheets}

As a first step we identify the various Riemann sheets by their value of $y(0)$ : the sheet labeled $s$ will have $y(0)=Y_{s}$ for that sheet. Obviously, $y(0)=0$ is a solution with

multiplicity $m$. In general, there will be $d_{P}$ solutions if $Q(y)=0$, and infinitely many if $Q$ is non-vanishing. It will be helpful if we can identify the Riemann sheet on which pairs 


\begin{tabular}{|l|c|}
\hline$s$ & $Y_{s} / \pi$ \\
\hline 1 & $(0.0000,0.0000)$ \\
3 & $(0.6649,2.3751)$ \\
5 & $(0.8480,4.4178)$ \\
7 & $(0.9633,6.4374)$ \\
9 & $(1.0478,8.4490)$ \\
11 & $(1.1145,10.4567)$ \\
\hline
\end{tabular}

Table 1: The first few Riemann sheet solutions for $F_{1}\left(Y_{s}\right)=0$.

$(x, y(x))$ lie when $x$ is small but nonzero. This is indeed possible, and we shall illustrate it using $F_{1}$. Let us write $y=\xi+i \eta$ with $\xi$ and $\eta$ real numbers. We are then looking for solutions of $F_{1}(\xi+i \eta)=0$, or

$$
\xi=\log \left(\frac{\eta}{\sin \eta}\right) \quad, \quad 1+\log \left(\frac{\eta}{\sin \eta}\right)-\frac{\eta}{\tan \eta}=0 .
$$

Inspecting the left-hand side of the last equation, we can immediately see that its zeroes are quite nicely distributed. We can usefully enumerate them as $\operatorname{Im}\left(Y_{s}\right)=u_{s}$, where the sheet number $s$ takes only the odd integer values $\pm 1, \pm 3, \pm 5, \ldots$ For positive $s$, the zero $u_{s}$ is certainly located in the interval where $\sin u_{s}>0$, i.e. $(s-1) \pi \leq u_{s}<s \pi$, and $u_{-s}=-u_{s}$. We have $u_{1}=u_{-1}=0$, and for increasing $s$ the zero $u_{s}$ moves upwards in its interval, until asymptotically we have $u_{s} \sim a_{s}-\left(\log a_{s}\right) / a_{s}$ with $a_{s}=(s-1 / 2) \pi$. In Tab.1 we give the values of $Y_{s}$ for $F_{1}$, for the first few values of $s$. Because the values $Y_{s}$ fall in disjoint intervals, for small $x$ we need to know $y(x)$ only to a limited accuracy in order to be able to identify its Riemann sheet. The only nontrivial case is that of sheets -1 and 1 , where it is sufficient to consider the complex $\operatorname{arguments:~for~} \arg (x)-\arg (y)=0$ we are on sheet 1, for $|\arg (x)-\arg (y)|=\pi$ we are on sheet -1 . Again, limited computer accuracy is acceptable here, and for larger $m$ we simply have $m$ different values of the argument, distinguished in an analogous manner. Note that of course the labeling of the sheets is rather arbitrary: we have chosen the odd integers in order to emphasize that both sheet 1 and -1 can be considered the principal Riemann sheet. For the bootstrap equation (3) it is more natural to label the single principal Riemann sheet with $y(0)=0$ as sheet number zero.

\section{Series expansion}

We want to compute $y(x)$ as a Taylor series around $x=0$ :

$$
y(x)=\sum_{n \geq 0} \alpha_{n} x^{n} .
$$


Obviously, $\alpha_{0}$ can be chosen as one of the $u_{s}$ above. On principal sheets, with $\alpha_{0}=0$, we also have immediately that $\alpha_{1}$ must be chosen out of the $m$ possibilities with $\alpha_{1}^{m}=1$. The other coefficients must then be computed (algebraically or numerically) by some recursive method, which we shall now discuss.

It would be straightforward to plug the expansion (7) into Eq. (2) and equate the powers of $x$ on both sides, but notice that, for $Q$ non-vanishing, the number of possible products of coefficients grows very rapidly, so that the computer time needed to find the first $N$ coefficients grows exponentially with $N$. As already mentioned in [3], the better way is to differentiate Eq. (2) with respect to $x$ so that we obtain the nonlinear differential equation

$$
y^{\prime}(x)\left[P^{\prime}(y) Q(y)+\left(Q(y)+Q^{\prime}(y)\right)\left(x^{m}-P(y)\right)\right]=m x^{m-1} Q(y) .
$$

This equation yields a recursion relation involving products of at most $d_{P}+d_{Q}+1$ coefficients, so that a truncated power series can be computed in polynomial time. As an example, for $F_{1}$ we find the following differential equation:

$$
y^{\prime}(x)\left(x^{2}+2 y(x)\right)=2 x,
$$

and the following recursion relation:

$$
\begin{aligned}
& \alpha_{0} \alpha_{1}=0 \quad, \quad 2 \alpha_{0} \alpha_{2}+\alpha_{1}^{2}-1=0 \\
& n \alpha_{0} \alpha_{n}+(n-2) \alpha_{n-2}+2 \sum_{p=1}^{n-1} p \alpha_{p} \alpha_{n-p}=0 \quad, \quad n \geq 3 .
\end{aligned}
$$

We see immediately that $y(x)$ is necessarily even in $x$ if $\alpha_{0} \neq 0$, i.e. on the non-principal Riemann sheets. In that case, we also see that if $\alpha_{n}, n=0,2, \ldots$ is a solution, then also $\alpha_{n}^{*}, n=0,2, \ldots$ is a solution, where the asterix stands for complex conjugation. This is a result of the fact that if $y(x)$ is a solution of Eq. (5), then also $y^{*}\left(x^{*}\right)$ is a solution. In practice, these solutions give the function values on the different Riemann sheets of one solution. The analysis of the previous section proves that $y_{s}(0)=y_{-s}(0)^{*}$ so that the solutions satisfy $y_{s}^{*}(x)=y_{-s}\left(x^{*}\right)$ and the expansion coefficients satisfy

$$
\alpha_{n}^{(s)}=\left(\alpha_{n}^{(-s)}\right)^{*} \text {. }
$$

On the principal Riemann sheets we have $\alpha_{0}=0$ and $\alpha_{1}^{2}=1$ as mentioned, and the two solutions on sheet 1 and sheet -1 are related by $y_{-1}(x)=y_{1}(-x)$. For $y_{1}(x)$ we find, finally:

$$
\alpha_{n}=-\frac{1}{2(n+1)}\left[(n-1) \alpha_{n-1}+2 \sum_{p=2}^{n-1} p \alpha_{p} \alpha_{n+1-p}\right],
$$

for $n \geq 2$. Using this relation we have been able to compute many thousands of terms. The recursion appears to be stable in the forward direction, but we have not tried to prove this or examine the stability in the general case.

In series expansions it is of course always important to know the convergence properties or, equivalently, the asymptotic behavior of $\alpha_{n}$ as $n$ becomes very large. In the next section, we therefore turn to the singularity structure of $y(x)$. 


\section{Singularities and branches}

In order to find information about the singularity structure of $y(x)$, we employ the techniques developed in [1], which we recapitulate here. Singularities are situated at those values $y_{k}$ of $y$ where

$$
F^{\prime}\left(y_{k}\right)=0 \text {. }
$$

Since $F$ is entire we also know that these singular points must form an enumerable set, i.e. we can find, and label, them as distinct points. We shall assume that these singularities are square-root branch points, for which it is necessary that

$$
F^{\prime \prime}\left(y_{k}\right) \neq 0
$$

If $F^{\prime \prime}$ vanishes at $y_{k}$ but $F^{\prime \prime \prime}$ does not, we have a cube-root branch point, and so on. If, for non-vanishing $Q$, all derivatives vanish (as for instance when $F(y)=e^{y}$ ) we have, of course, a logarithmic branch point. We know that $y=-\infty$ corresponds to a logarithmic branch point, and it is to remove this to infinity in the $x$ plane that we have required $d_{P}>0$. In our examples all the singularities at finite $x$ will be square-root branch points. The position of the singularity in the $x$ plane, $x_{k}$, is of course given by

$$
F\left(y_{k}\right)=x_{k}^{m},
$$

so that there are $m$ different possible positions, lying equally spaced on a circle around the origin. We shall denote them by $x_{k, p}$ with $p=1,2, \ldots, m$. Note that, in first instance, it is not clear at all whether $x_{k, p}$ for certain $k$ and $p$ is indeed a singular point on a specific Riemann sheet. Later on, we shall describe how to determine this numerically. For values of $x$ close to an observed singular point $x_{k, p}$ we may expand the left-hand and right-hand side of Eq. (目) to obtain

$$
\frac{1}{2}\left(y-y_{k}\right)^{2} F^{\prime \prime}\left(y_{k}\right) \sim m F\left(y_{k}\right)\left(\frac{x}{x_{k, p}}-1\right),
$$

where we have dropped the higher derivative terms. Very close to the branch point we may therefore approximate $y(x)$ by

$$
y(x) \sim y_{k}+\beta_{k, p}\left(1-\frac{x}{x_{k, p}}\right)^{1 / 2}, \quad \beta_{k, p}^{2} \equiv-\frac{2 m F\left(y_{k}\right)}{F^{\prime \prime}\left(y_{k}\right)} .
$$

Note that there are only two possible values for $\beta_{k, p}$, and each singular point $x_{k, p}$ goes with one or the other of these. Again numerical methods will help in determining which one of the two is the correct choice.

We are now in a position to compute the asymptotic behavior of the coefficients $\alpha_{n}$. To find it, we first determine, for a given Riemann sheet, which are the $x_{k, p}$ that lie closest to the origin: this gives us the radius of convergence of the expansion of $y(x)$ in that Riemann 
sheet. We then have to determine those $p$ for which $x_{k, p}$ is actually a singular point. We shall do this numerically, in the way described in the following section. Let us denote the set of values of $p$ for which this is the case by $\mathcal{P}$. Now, we may use the fact that

$$
\begin{aligned}
& \sqrt{1-x}=1-\sum_{n \geq 1} \gamma_{n} x^{n} \quad, \quad \gamma_{n}=\frac{(2 n-2) !}{2^{2 n-1}(n-1) ! n !}, \\
& \gamma_{n} \sim \frac{1}{\sqrt{4 \pi}} n^{-3 / 2}+\mathcal{O}\left(n^{-5 / 2}\right) \quad, \quad n \rightarrow \infty
\end{aligned}
$$

where we have chosen that square root that is real and positive for $1-x$ real and positive. The asymptotic behavior of $\alpha_{n}$ as $n \rightarrow \infty$ must therefore be given by

$$
\alpha_{n} \sim \frac{-1}{n^{3 / 2} \sqrt{4 \pi}} \sum_{p \in \mathcal{P}} \frac{\beta_{k, p}}{x_{k, p}^{n}} .
$$

Amongst other things, this provides a powerful numerical check on the accuracy of the $\alpha_{n}$ as computed by the recursive technique. We shall now discuss how the singularity structure of our problem can be investigated numerically.

\section{Computer searches for sheet structures}

The main tool we use for our computer studies is a method for taking small steps over a Riemann sheet, that is, given the fact that for some value $x_{1}$ the point $y_{1}=y\left(x_{1}\right)$ is determined to belong to a certain Riemann sheet, we perform a small step $\Delta x$ to a point $x_{2}$ and find the point $y_{2}=y\left(x_{2}\right)$ on the same Riemann sheet. Our method to do this is nothing but Newton-Raphson iteration: we simply iterate the mapping

$$
y \leftarrow y-\frac{F(y)-x_{2}^{m}}{F^{\prime}(y)},
$$

until satisfactory convergence is obtained. The starting value for this iteration is just the point $y_{1}$. A few remarks are in order here. In the first place, it must be noted that for this method to work, $y_{1}$ must be in the basin of attraction of $y_{2}$. Since, except at the branch points, which we shall expressly avoid, $y(x)$ is a continuous and differentiable function of $x$, this can always be arranged by taking $\Delta x$ small enough. In the second place, the accuracy with which $y_{1}$ is actually a solution of Eq. (2) is not important as long as it is in the basin of attraction of $y_{2}$ : therefore, there is no buildup of numerical errors in this method if we restrict ourselves to just keeping track of which Riemann sheet we are on. Finally, problems could arise if two Riemann sheet values of $y$ for the same $x$ are very close. But, since $F$ is an entire function, we know that the solutions of Eq. (2) must either completely coincide or be separated by a finite distance, any inadvertent jump from one sheet to another can be detected and cured by, again, taking a small enough $\Delta x$.

We have applied the following method for detecting and characterizing the various singular points. We start on a Riemann sheet $s_{1}$ at a value $x$ close to zero, and determine 
$y(x)$ on that Riemann sheet. We then let the parameter $x$ follow a prescribed contour that circles a selected would-be singularity $x_{k, p}$ once (and no other singularities), and then returns to the starting point close to the origin. We then determine to which Riemann sheet the resulting $y$ belongs. In this way we can find whether $x_{k, p}$ is, in fact, a singular point for the starting sheet, and, if so, which two sheets are connected there. It is also possible, of course, to certify the square-root branch point nature of a singular point by circling twice around it, and checking that one returns to the original Riemann sheet.

One important remark is in order here. In our tracking over the Riemann sheet, it is necessary that we do not cross branch cuts (except of course the one connected to the putative singularity). Since these branch cuts can be moved around in the complex $x$ plane, the contour chosen defines the (relative) position of the branch cuts. The sheets that are said to be connected at a particular branch cut are therefore also determined by the choice of contour. Of course, choosing a different contour will change the whole system of interconnected sheets in a consistent manner, so that in fact, given one choice of contour and its system of sheets, we can work out what system of sheets will correspond to another choice of contour. We shall illustrate this in the following.

Suppose, now, that $x_{k, p}$ is one of the singular points on a certain sheet that is closest to the origin. We can then follow, on that sheet, a straight line running from $x_{1}$ close to the origin to a point $x_{2}$ for which $x_{2} / x_{k, p}$ is real and just a bit smaller than one. Since $x_{k, p}$ is by assumption closest to the origin, there is then no ambiguity involved in determining which one of the two possible complex arguments of $\beta_{k, p}$ we have to take. Thus, we can find all the information needed to compute the asymptotic behavior of $\alpha_{n}$ on that sheet.

\section{An example}

Having established the necessary machinery, we shall now discuss a concrete example of our method. For this, we have taken the function $F_{1}$ of Eq. (5). It is, in fact, closely related to the very well-understood bootstrap equation (3): by substituting, in Eq. (3), $y \rightarrow \log 2+y$ and $x \rightarrow 2 \log 2-1-x^{2}$, we obtain Eq. (5). Its Riemann sheet structure, however, is quite different, as we shall see. Note that under this transformation, the origin $x=0, y=0$ for $F_{1}$ corresponds to the first singularity in $F_{B}$.

\subsection{The singularities}

The values of $y(0)$ on the different Riemann sheets for $F_{1}$, namely $Y_{s}$ for $s= \pm 1, \pm 3, \ldots$ have already been discussed above. The singular values $y_{k}$ are simply given by

$$
F_{1}^{\prime}\left(y_{k}\right)=2 e^{y_{k}}-2=0 \quad \Rightarrow \quad y_{k}=2 i \pi k
$$

so that the possible singular points $x_{k, p}$ satisfy

$$
x_{k, p}^{2}=-4 i \pi k
$$


Note that $k=0$ does not correspond to a singular point. The positions of the possible singularities in the complex $x$ plane are therefore as follows. For positive integer $k$ :

$$
\begin{aligned}
& x_{k, 1}=i z_{k} \quad, \quad x_{k, 2}=-i z_{k}, \\
& x_{-k, 1}=z_{k} \quad, \quad x_{-k, 2}=-z_{k} \\
& z_{k}=(1+i) \sqrt{2 \pi k} .
\end{aligned}
$$

At all these various possible singularities, we have

$$
\beta_{k, p}^{2}=8 i \pi k
$$

and therefore we may write

$$
\begin{array}{ll}
\text { for } k>0: & \beta_{k, p}=\epsilon_{k, p}(1+i) \sqrt{4 \pi|k|}, \\
\text { for } k<0: & \beta_{k, p}=\epsilon_{k, p}(1-i) \sqrt{4 \pi|k|},
\end{array}
$$

where the only number to be determined is $\epsilon_{k, p} \in\{-1,1\}$. It must be kept in mind that the value of $\epsilon$ depends of course on the sheet: we take the convention that we work on the sheet with the lowest number (in absolute value). When viewed from the other sheet, the value of $\epsilon$ is simply opposite.

\subsection{The Riemann sheet structure}

We now have to discuss how the branch cuts should run in the complex $x$ plane. There are two simple options (and an infinity of more complicated ones): in the first option (I), we choose to let the branch cuts extend away from the origin parallel to the real axis. This corresponds to tracking a contour that, say, first moves in the imaginary direction, and then in the real direction, to arrive close to the chosen singularity. The other option (II) is to take the cuts parallel to the imaginary axis, so that a contour that does not cross branch cuts en route first goes in the real direction, and then in the imaginary direction. Note that these two alternatives do, indeed, correspond to different implied relative positionings of the branch cuts. In Fig. 1.b we show the contour used in examining singularity $x_{2,1}$ under option I. The contour starts on sheet number 1 close to the origin (so that $y$ is close to $Y_{1}$ ), moves upwards and then to the left, circles the singularity once anti-clockwise, and returns to its starting point by the same route in order to enable us to determine the resulting Riemann sheet number. Fig. 1. 1.c shows the corresponding path in the $y$ plane. It ends again close to $Y_{1}$ so that, for this choice of contour and its induced branch structure (indicated in the figure), sheet 1 does not have a branch point at $x_{2,1}$. Fig. 1. d shows what happens if, instead of sheet number 1 , we start at sheet number 3: the $y$ track starts then at close to $Y_{3}$, but ends up close to $Y_{5}$, so that we conclude that sheets 3 and 5 are connected at $x_{2,1}$. If we run through the whole contour twice, we get the $y$ track presented in Fig. 1.e, where the $y$ track ends up again at $Y_{3}$ as expected for a square root branch cut. Under option II, we rather use the contour indicated in Fig.2.a, which first moves to the left and 


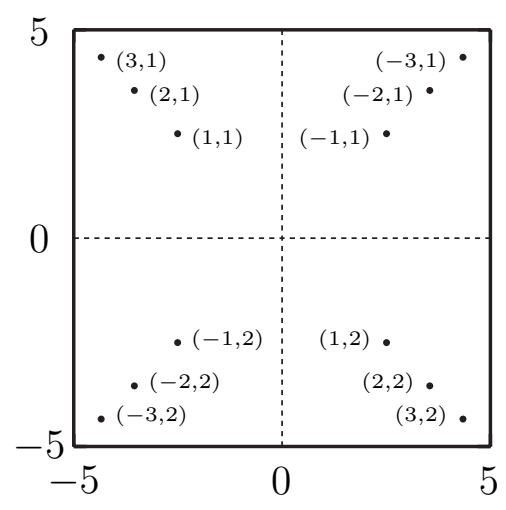

a: The numbering $(k, p)$ of the singularities.

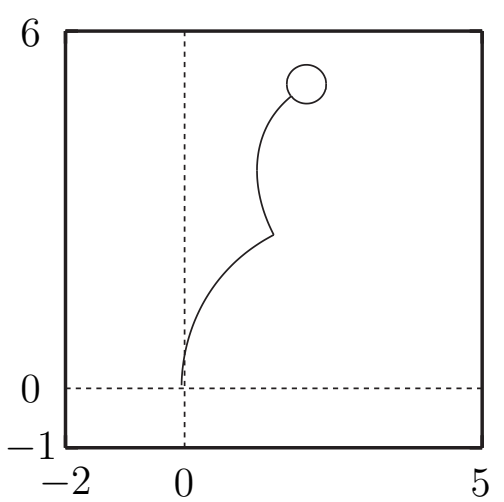

c: sheet 1 , single loop

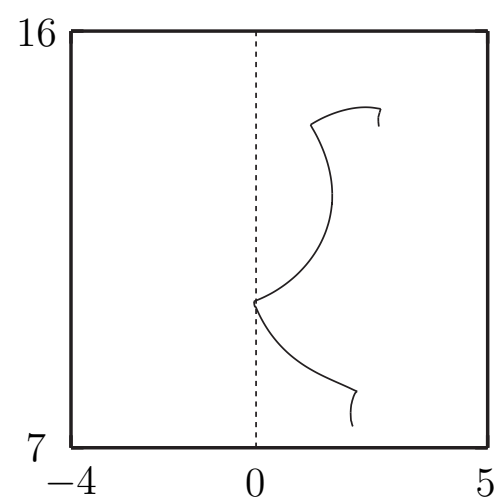

d: sheet 3 , single loop

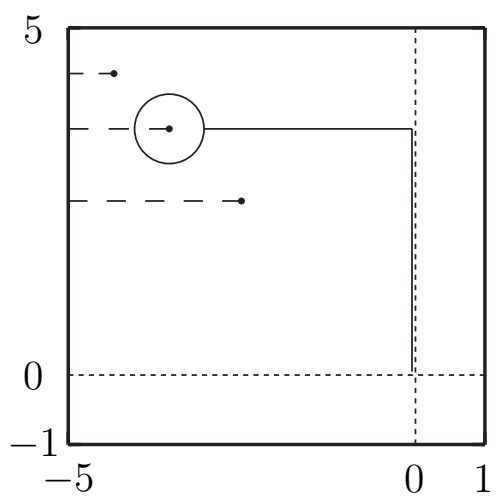

$\mathrm{b}: x$-plane

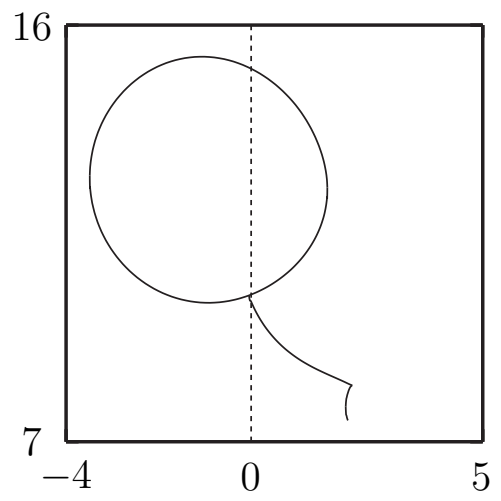

e: sheet 3 , double loop

Figure 1: The numbering $(k, p)$ of the singularities, and loops around $x_{2,1}$ under option I.

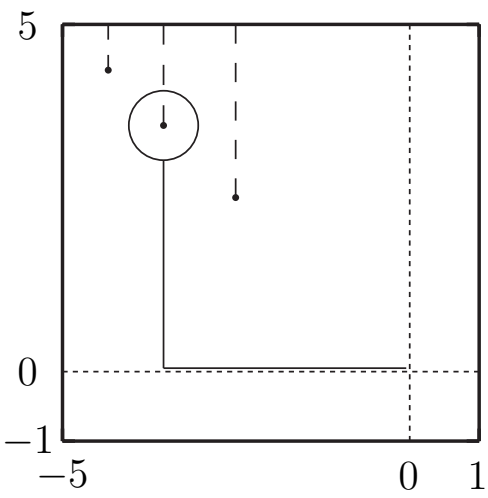

a: $x$-plane

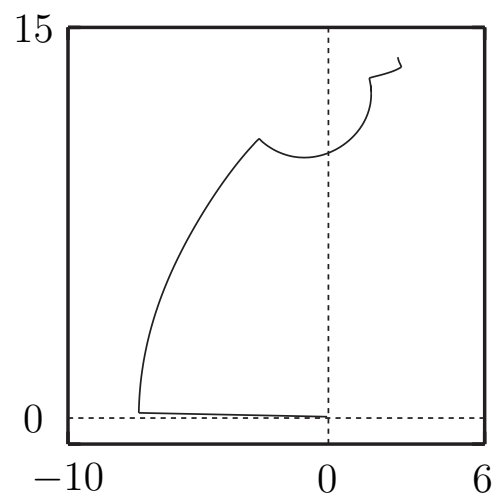

b: sheet 1 , single loop

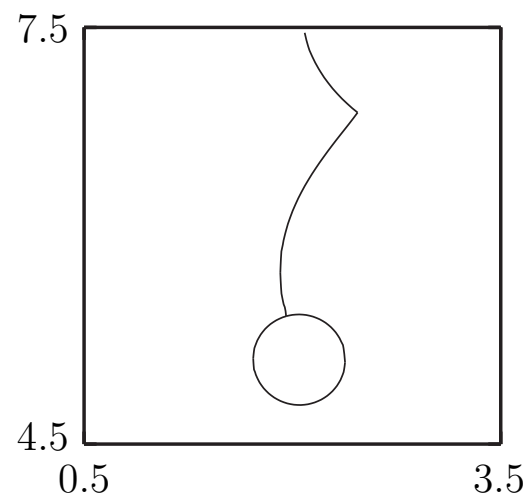

c: sheet 3 , single loop

Figure 2: Loops around $x_{2,1}$ under option II. 


\begin{tabular}{|c|c|c|c|c|c|c|c|c|}
\hline$k$ & $x_{k, 1}$ & $\epsilon_{k, 1}$ & $x_{k, 2}$ & $\epsilon_{k, 2}$ & $x_{-k, 1}$ & $\epsilon_{-k, 1}$ & $x_{-k, 2}$ & $\epsilon_{-k, 2}$ \\
\hline 1 & $(1,3)$ & -1 & $(-1,3)$ & -1 & $(-1,-3)$ & -1 & $(1,-3)$ & -1 \\
2 & $(3,5)$ & -1 & $(3,5)$ & -1 & $(-3,-5)$ & -1 & $(-3,-5)$ & -1 \\
3 & $(5,7)$ & -1 & $(5,7)$ & -1 & $(-5,-7)$ & -1 & $(-5,-7)$ & -1 \\
4 & $(7,9)$ & -1 & $(7,9)$ & -1 & $(-7,-9)$ & -1 & $(-7,-9)$ & -1 \\
5 & $(9,11)$ & -1 & $(9,11)$ & -1 & $(-9,-11)$ & -1 & $(-9,-11)$ & -1 \\
\hline
\end{tabular}

Table 2: Sheets connected at the first few singularities (option I), and the corresponding value for $\epsilon$.

\begin{tabular}{|c|c|c|c|c|c|c|c|c|}
\hline$k$ & $x_{k, 1}$ & $\epsilon_{k, 1}$ & $x_{k, 2}$ & $\epsilon_{k, 2}$ & $x_{-k, 1}$ & $\epsilon_{-k, 1}$ & $x_{-k, 2}$ & $\epsilon_{-k, 2}$ \\
\hline 1 & $(1,3)$ & -1 & $(-1,3)$ & -1 & $(-1,-3)$ & -1 & $(1,-3)$ & -1 \\
2 & $(1,5)$ & -1 & $(-1,5)$ & -1 & $(-1,-5)$ & -1 & $(1,-5)$ & -1 \\
3 & $(1,7)$ & -1 & $(-1,7)$ & -1 & $(-1,-7)$ & -1 & $(1,-7)$ & -1 \\
4 & $(1,9)$ & -1 & $(-1,9)$ & -1 & $(-1,-9)$ & -1 & $(1,-9)$ & -1 \\
5 & $(1,11)$ & -1 & $(-1,11)$ & -1 & $(-1,-11)$ & -1 & $(1,-11)$ & -1 \\
\hline
\end{tabular}

Table 3: Sheets connected at the first few singularities (option II), and the corresponding value for $\epsilon$.

then upwards. Fig.2.b shows the resulting $y$ path, which does not return to $Y_{1}$ but rather to $Y_{5}$, indicating that under this choice of contour the sheets labeled 1 and 5 are connected at $x_{2,1}$. Fig. 2.c shows that, now, sheet 3 is insensitive to this singularity.

In this way we have mapped the various singularities around the origin. In Tab. 2 a we present the pairs of sheets that are pairwise connected at the first few singularities, under option I, and the observed value for $\epsilon$, which turns out to be -1 in all cases. We point out that at each singularity only two sheets out of all infinitely many are connected. Note the somewhat atypical situation at the lowest-lying singularities $x_{1, \pm 1}$ and $x_{-1, \pm 1}$. The alternative option II results in Tab. 3. Note that the higher-lying singularities now show a sheet structure similar to the lowest ones. In fact, this is the choice that corresponds most directly to the analysis of the sheet structure of the bootstrap equation in [3], with of course the extra complication in the fact that the bootstrap equation (3) has $m=1$ while for $F_{1}, m=2$. Note that, once again, $\epsilon=-1$ in all cases.

\subsection{Asymptotic behavior of the series expansion coefficients}

We shall now illustrate how the information on the $x_{k, p}$ and $\beta_{k, p}$ allows us to compute the asymptotic behavior of the series expansion coefficients $\alpha_{n}$. 


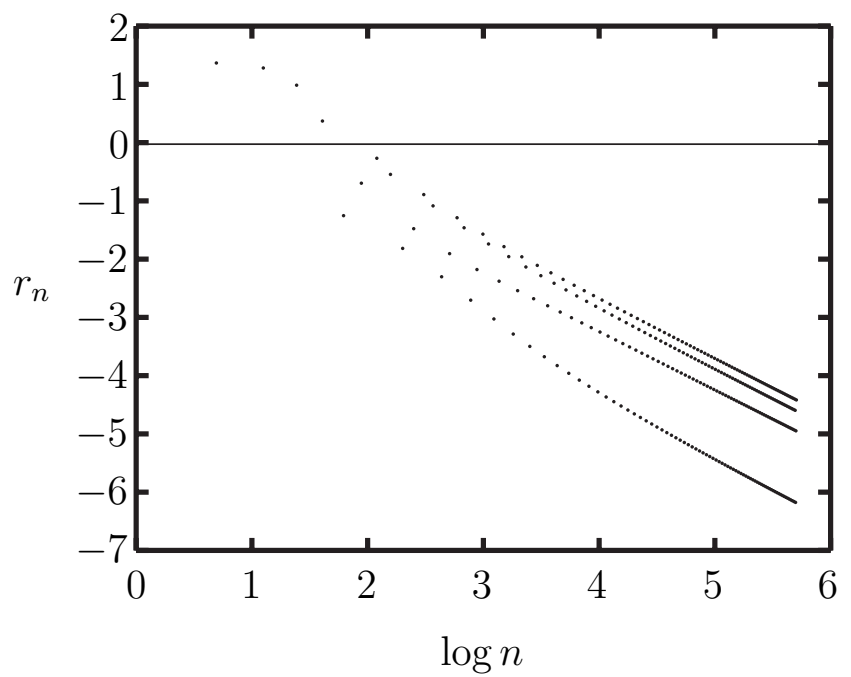

Figure 3: $r_{n}$, defined in Eq. (28), as function of $\log n$.

First Riemann sheet. In this sheet, the singularities closest to the origin, and their corresponding $\beta$ 's are

$$
\begin{aligned}
& x_{1,1}=\sqrt{4 \pi} \exp (3 i \pi / 4), \quad \beta_{1,1}=\sqrt{8 \pi} \exp (-3 i \pi / 4), \\
& x_{-1,2}=\sqrt{4 \pi} \exp (-3 i \pi / 4) \quad, \quad \beta_{-1,2}=\sqrt{8 \pi} \exp (3 i \pi / 4) .
\end{aligned}
$$

Using Eq. (19), we see that the asymptotic form of the coefficients on sheet 1 is given by

$$
\begin{aligned}
& \alpha_{n}^{(1)} \sim \alpha_{n}^{\text {asym }}, \\
& \alpha_{n}^{\text {asym }}=\frac{2}{n^{3 / 2}(4 \pi)^{n / 2}} c_{n}, \\
& c_{n}=-\sqrt{2} \cos \left(\frac{3 n \pi}{4}+\frac{3 \pi}{4}\right)= \begin{cases}(-)^{p} & n=4 p \\
0 & n=4 p+1 \\
(-)^{p+1} & n=4 p+2 \\
(-)^{p} \sqrt{2} & n=4 p+3\end{cases}
\end{aligned}
$$

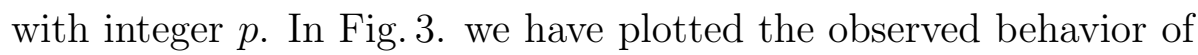

$$
r_{n}=\log \left(\frac{(4 \pi)^{n / 2} n^{3 / 2}}{2}\left|\alpha_{n}-\alpha_{n}^{\text {asym }}\right|\right)
$$

on the first Riemann sheet, against $\log n$. The coefficients clearly converge to the computed behavior, and we can even distinguish that the leading corrections go as $n^{-5 / 2}$; the four separate lines that emerge are just the four different forms of $c_{n}$. The series expansion for Riemann sheet -1 are simply obtained from

$$
\alpha_{n}^{(-1)}=(-)^{n} \alpha_{n}^{(1)} .
$$


Higher Riemann sheets. We first consider positive sheet label $s=3,5,7, \ldots$ and put $k=(s-1) / 2$. We then have

$$
x_{k, 1}=-x_{k, 2}=\sqrt{4 \pi k} \exp (i \pi / 4) \quad, \quad \beta_{k, 1}=\beta_{k, 2}=(1+i) \sqrt{4 \pi k} .
$$

As we have already seen $\alpha_{n}$ vanishes for odd $n$, and for even $n$ we have the following asymptotic form:

$$
\alpha_{4 p}^{(s)} \sim \frac{2(1+i) \sqrt{k}}{(4 \pi k)^{2 p}}(-)^{p+1}, \quad \alpha_{4 p+2}^{(s)} \sim \frac{2(1-i) \sqrt{k}}{(4 \pi k)^{2 p+1}}(-)^{p},
$$

for integer $p$. For negative $s$, we use Eq. (11), which also holds asymptotically.

\section{Conclusion}

We have shown how numerical tracking can be helpful in the investigation of the Riemann sheet structure of the solution of certain algebraic complex equations. Furthermore, we have shown how the series expansions around the origin on the different sheets and the asymptotic behavior of their coefficients can be determined. The results of the numerical analyses have been justified by the fact that only finite computer accuracy was required in the specific calculations.

\section{References}

[1] E.N. Argyres, R. Kleiss, C.G. Papadopoulos, Amplitude estimates for multi-Higgs production at high-energies, Nucl. Phys. B391:42-56 (1993);

[2] A. van Hameren and R. Kleiss, Quantum field theory for discrepancies, in preparation.

[3] R. Hagedorn and J. Rafelski, Analytic Structure and Explicit Solution of an Important Implicit Equation, Commun. Math. Phys. 83:563-578 (1982).

[4] A.S. Kapoyannis, C.N. Ktorides, A.D. Panagiotou, An extension of the Statistical Bootstrap Model to include Strangeness, J. Phys. G23:1921 (1997).

[5] N.N. Khuri and O.A. McBryan, Explicit solutions for the 't Hooft transformation, Phys. Ref. D20:881-886 (1979). 